Meta

Journal des traducteurs

Translators' Journal

\title{
L'alternance passé-présent dans le récit : contraintes de la traduction du français vers l'anglais
}

\section{Hélène Chuquet}

Volume 45, numéro 2, juin 2000

URI : https://id.erudit.org/iderudit/002245ar

DOI : https://doi.org/10.7202/002245ar

Aller au sommaire du numéro

\section{Éditeur(s)}

Les Presses de l'Université de Montréal

ISSN

0026-0452 (imprimé)

1492-1421 (numérique)

Découvrir la revue

Citer cet article

Chuquet, H. (2000). L'alternance passé-présent dans le récit : contraintes de la traduction du français vers l'anglais. Meta, 45(2), 249-262.

https://doi.org/10.7202/002245ar
Résumé de l'article

Cet article examine la question des choix de traduction à effectuer dans le passage du français à l'anglais lorsque l'on se trouve en présence de récits ayant recours à l'alternance temporelle entre temps du passé et présent dit « de narration ». Quelques exemples empruntés à la presse et à la fiction en français, puis en anglais, montreront que le phénomène de l'alternance temporelle se rencontre dans les deux langues, mais qu'il n'obéit pas aux mêmes contraintes. Une analyse du mode de construction du récit au présent fondée sur le concept de repérage emprunté à la théorie d'Antoine Culioli permet de dégager un certain nombre de critères destinés à guider les choix de traduction.L'étude d'une dernière série d'extraits dont la langue de départ est le français vise àappliquer les hypothèses théoriques avancées à la pédagogie de la traduction, en vue de proposer quelques conseils susceptibles d'être donnés à des étudiants. 


\title{
L'alternance passé-présent dans le récit: contraintes de traduction du français vers l'anglais
}

\author{
HÉLÈNE CHUQUET \\ FORELL, MSHS, Université de Poitiers, Poitiers, France
}

\begin{abstract}
RÉSUMÉ
Cet article examine la question des choix de traduction à effectuer dans le passage du français à l'anglais lorsque l'on se trouve en présence de récits ayant recours à l'alternance temporelle entre temps du passé et présent dit «de narration». Quelques exemples empruntés à la presse et à la fiction en français, puis en anglais, montreront que le phénomène de l'alternance temporelle se rencontre dans les deux langues, mais qu'il n'obéit pas aux mêmes contraintes. Une analyse du mode de construction du récit au présent fondée sur le concept de repérage emprunté à la théorie d'Antoine Culioli permet de dégager un certain nombre de critères destinés à guider les choix de traduction. L'étude d'une dernière série d'extraits dont la langue de départ est le français vise à appliquer les hypothèses théoriques avancées à la pédagogie de la traduction, en vue de proposer quelques conseils susceptibles d'être donnés à des étudiants.
\end{abstract}

\section{ABSTRACT}

This article looks at choices inherent in French to English translation of narrative texts in which past tenses as well as the so-called "narrative present tense" are used. Examples from newspaper articles and excerpts of fiction in both French and English show that the alternating use of tenses is prevalent in both languages, but that different restrictions apply. Culioli's concept of "repérage" serves as a basis for analyzing the present tense construction of the narrative text, providing some criteria for guiding translation choices. Another set of texts, originally in French, is used to demonstrate hypotheses underlying translation pedagogy in an attempt to establish guidelines for students.

\section{MOTS-CLÉS/KEYWORDS}

présent et passé de narration, traduction du français vers l'anglais, alternance temporelle, repérage, pédagogie de la traduction

Le but de cette étude est avant tout de présenter quelques applications possibles à la pédagogie de la traduction de recherches que j'ai menées il y a quelques temps sur le présent dit «de narration» en anglais et en français (voir Chuquet 1994). Plus spécifiquement, je voudrais me pencher sur les problèmes posés par l'alternance dans les récits entre plusieurs temps de la narration (principalement entre le présent et passé), par cette hétérogénéité temporelle que l'on rencontre souvent, en particulier en français, qui a été étudiée par Dolz (1993) sous le nom de "ruptures temporelles» et est désignée en anglais sous le nom de tense-switching.

Les textes littéraires et journalistiques français contemporains font un grand usage de l'hétérogénéité temporelle, et tout pratiquant de la traduction - apprenti ou non - est amené à se poser la question de savoir si l'on doit ou si l'on peut maintenir ces ruptures en passant du français à l'anglais. Si oui, en vertu de quels 
critères? Quelles instructions fiables, linguistiquement fondées, peut-on donner à des étudiants... autres que le conseil classique, souvent rencontré dans les manuels de traduction en France, de traduire un présent historique français par un prétérit anglais ${ }^{1}$.

Il est vrai que l'on a le sentiment d'une plus grande résistance à l'hétérogénéité temporelle en anglais qu'en français. De nombreux auteurs notent que le présent de narration est moins utilisé en anglais (Bellos 1978; Comrie 1976; Fleischman 1990), et les locuteurs anglophones y sont souvent très réticents, soit lorsqu'on leur demande de traduire spontanément un passage français comportant des variations temporelles, soit lorsqu'on leur soumet des exemples d'hétérogénéité en anglais. Ceci est vrai tout au moins en ce qui concerne l'écrit, car le tense-switching dans le récit oral est largement représenté dans les deux langues, et bien documenté en anglais, notamment nord-américain (cf. Schiffrin 1981; Wolfson 1982).

Il n'en reste pas moins que l'on est en présence d'un paradoxe - c'est d'ailleurs ce qui m'avait poussée à étudier la question du présent de narration d'un point de vue contrastif. De nombreuses œuvres littéraires en anglais (de Chaucer aux auteurs contemporains, en passant par Defoe ou Dickens, pour ne citer que quelques exemples) ont recours à l'alternance présent/prétérit dans le récit ${ }^{2}$ : cela existe donc, alors pourquoi cette «censure» du traducteur du français vers l'anglais? Mais en même temps, l'observation de traductions anglaises de romans français contemporains révèle que, lorsqu'est calquée l'hétérogénéité temporelle, cela pose parfois des problèmes de lisibilité et cela n'est pas ressenti comme naturel par le lecteur. Je me suis intéressée, par exemple, aux traductions de plusieurs romans de Patrick Modiano, auteur qui joue beaucoup sur les variations temporelles, notamment entre le récit au présent et au passé composé. Tous les traducteurs ne font pas le même choix: la traductrice du roman Les boulevards de ceinture, Catherine Hillier, maintient quasiintégralement en anglais l'organisation temporelle du français; à l'inverse, la traductrice de Quartier perdu, Anthea Bell, a transposé la totalité du récit au prétérit. Dans le premier cas, l'effet produit dans la langue d'arrivée est très variable selon les passages, et heurte fréquemment le lecteur anglophone; dans le second, le roman se lit parfaitement d'un bout à l'autre en anglais, mais la comparaison avec l'original fait apparaitre une perte indéniable.

J'ai donc voulu voir si une étude linguistique du fonctionnement du récit au présent dans les deux langues permettait d'apporter certaines explications à ce phénomène et de donner des critères pour choisir en connaissance de cause. Il m'est apparu qu'en fait cette question de l'alternance temporelle et des contraintes auxquelles elle obéit est étroitement liée au mode de construction du récit permettant l'emploi du présent dans chacune des langues. Pour essayer de le montrer, je partirai de quelques exemples de récits à repérage temporel variable dans chaque langue, car en définitive c'est l'observation des choix énonciatifs originaux et des restrictions d'emploi rencontrées qui permet au linguiste/à l'enseignant/au traducteur de se donner, dans une certaine mesure, des critères objectifs de choix.

\section{Exemples d'hétérogénéité temporelle en français}

Les extraits (1) à (7) ci-dessous montrent que l'hétérogénéité en français se rencontre dans tous les types de textes: journalistique, historique, littéraire, et quel que soit le type de récit: véridique objectif ou de fiction. 
(1) L'année dernière, alors que la Grèce s'engluait dans la boue des scandales politicofinanciers qui conduiront à la chute des socialistes, M. Caramanlis compare son pays à un «immense asile de fous».

(Le Monde, 6-7 mai 1990)

(2) Le 3 août 1980, un incendie se déclare dans les entrepôts du Pontel, à Villiers-SaintFrédéric, près de Rambouillet, où sont stockés une partie des trésors de la Cinémathèque. En quelques minutes, tout disparaît en fumée. On ne saura jamais exactement ce qui a été définitivement perdu. [...] Cette catastrophe s'est reproduite l'année suivante à Mexico, à une échelle encore plus impressionnante: toutes les réserves de la cinémathèque de la ville ont flambé en un temps record. Il y a deux ans, celle de Coblence, en RFA, connaît à son tour l'épreuve du feu.

(Le Monde, 19 avril 1990)

(3) C'est sur les eaux de cette formidable rade-abri que la flotte allemande se saborda le 21 juin 1919. [...] Dans la nuit du 14 octobre 1939, c'est encore sur la rade de ScapaFlow qu'un U-boot allemand envoie par le fond le cuirassé Royal Oak. [...] C'est à la suite de cette attaque que le gouvernement britannique décida de fermer pour de bon les passes Est...

(Le Monde Voyages, 31 mars 1990)

Ces trois premiers extraits sont caractéristiques des récits que l'on rencontre dans la presse française, avec la multiplication des repères et des variations temporelles. En (1) comme en (2), le repère temporel antéposé ( «l'année dernière », «le 3 août $1980 »)$ suffit à installer le récit dans le révolu, par rupture temporelle objective par rapport au moment de l'énonciation, et le renvoi aux événements situés dans ce plan décroché peut s'effectuer sans autre marquage de "passé» sur les formes verbales. C'est un type d'organisation temporelle que l'on rencontre fréquemment dans les récits de faits divers, les nécrologies, les rétrospectives de carrières etc. L’alternance privilégiée dans le récit journalistique de faits divers est celle entre présent et passé composé, telle qu'on l'observe en (2): la forme aspectuellement non marquée du présent sert à poser les événements bruts en relation avec le repère, tandis que le passé composé, à la fois aspect accompli du présent et temps du passé, est à la fois compatible avec le récit événementiel jalonné de dates révolues et avec le commentaire de ces mêmes événements à partir d'un point de vue énonciatif. L'alternance entre passé simple et présent illustrée en (3) se situe, elle, intégralement dans le plan du récit en rupture par rapport au moment de l'énonciation. Elle constitue une forme de structuration du récit, permettant de mettre en valeur la saillance de tel ou tel événement, de produire un effet d'ouverture, puis de clôture d'une séquence événementielle (voir à ce sujet Monville-Burston et Waugh 1985; Simonin 1984).

C'est cet effet de structuration du récit par les changements de focalisation dûs aux variations temporelles qui est souvent exploité dans les narrations historiques ouvrages d'histoire et, de façon encore plus frappante, manuels scolaires d'histoire ainsi que l'illustre l'extrait suivant:

(4) Dès 833, on voit les Hongrois, dont le nom apparaît alors pour la première fois, inquiéter les populations sédentaires aux environs de la mer d'Azov. Bientôt ils menacent à chaque instant de couper la route du Dniepr, en ce temps voie commerciale extrêmement active par où les esclaves achetés de toutes parts allaient s'échanger contre les marchandises. [...] Mais de nouvelles hordes sorties, après eux, de par delà l'Oural, les Petchénègues, les harcèlent sans cesse. Le chemin du sud leur est barré. [...] Ainsi refoulés, la plupart d'entre eux franchirent les Carpathes, vers 896 [...]. 
[...] Les tentatives des Scandinaves pour s'installer sur le sol britannique se dessinèrent dès leur premier hivernage: en 851, comme on l'a vu. Depuis lors, les bandes, se relayant plus ou moins entre elles, ne lâchent plus leur proie.

(Bloch 1939: 30, 48)

On notera ici le caractère assez systématique du rapport entre position du repère de localisation temporelle et choix du temps verbal: tantôt c'est le repère adverbial antéposé qui installe le plan du révolu dans lequel s'insère l'événement au présent, tantôt c'est le passé simple qui détermine l'événement comme appartenant au révolu, la date postposée n'ayant plus que le statut de commentaire, de précision supplémentaire.

Dans aucun de ces quatre extraits l'hétérogénéité temporelle ne peut de façon satisfaisante être maintenue en anglais. Le récit objectif, daté se fera au prétérit, tout entier construit en rupture temporelle par rapport au moment de l'énonciation. C'est d'ailleurs le choix qui est fait systématiquement dans les traductions de ce type d'articles du Monde paraissant dans le Guardian Weekly (extrait (2) par exemple). L'ouvrage de Marc Bloch d'où est tiré l'extrait (4) a, lui, été traduit en conservant presque intégralement l'hétérogénéité temporelle: cette traduction produit un effet peu naturel à la lecture, surtout si on la compare avec le texte original d'ouvrages historiques comparables rédigés en anglais qui, eux, ont recours de façon homogène aux temps du passé.

Les extraits (5) à (7) offrent des exemples tout à fait classiques d'alternance passé présent dans le récit littéraire en français:

(5) Pacôme, le maître jardinier, cherchait une institutrice pour sa fille Surette. [...] Il se préparait à partir pour l'Angleterre, afin d'en ramener la perle qui conviendrait à Surette, lorsqu'un soir, à minuit, on frappa à la porte de la maison.

La servante va ouvrir, poussée par son maître, car elle a peur. Elle revient enthousiaste, décrivant une jeune fille brune très jolie, entre dix-huit et vingt ans, qui se présente pour la place de gouvernante. On la fait entrer.

(Prassinos 1972: 79)

(6) Ce soir-là, le 17 août 1874, sept jours seulement après son vingt et unième anniversaire, Michel s'habille soigneusement en civil, baise sa cuirasse et son casque comme un moine près de se défroquer baiserait sa coule, et va prendre à la gare de Versailles ce train de Paris qui faillit être si fatal à son père. Depuis le retour de la paix, les passeports n'étaient plus un problème. Il monta gare Saint-Lazare dans un wagon pour Dieppe, d'où il s'embarqua pour l'Angleterre.

(Yourcenar 1977: 259)

(7) Il vida son bock d'un trait, ne remarquant son voisin qu'au moment où celui-ci se détachait du bar pour gagner la sortie. Cadin revint sur ses pas.

- Vous êtes facteur? Vous travaillez à la poste de Merville?

Son interlocuteur, un minuscule bonhomme aux joues couperosées, plaque ses paumes sur ses moustaches pour essuyer les traces de vin avant de répondre.

(Daeninckx 1984)

Tout se passe comme si, dans le cadre décroché de la fiction, le présent et le passé simple pouvaient alterner librement comme formes de la narration événementielle, construisant les procès comme vus de l'extérieur, envisagés en bloc sous l'aspect perfectif et insérés dans une séquence. Le passage d'une forme à l'autre a pour effet de créer des ruptures, des démarcations, qui peuvent être associées à des changements 
ou des transitions dans la trame narrative - changement d'actant et de localisation spatiale en (5), changement de lieu en (6), où l'on note le rôle de transition joué par l'énoncé statique à l'imparfait ${ }^{3}$, changement d'actant après la réplique de discours direct en (7). Cela dit, si l'on peut trouver des explications stylistiques à cette alternance temporelle, sur le plan linguistique, on a bien le sentiment que les deux formes effectuent la même opération, à ceci près que le repérage en rupture qui caractérise par force le récit de fiction n'est pas marqué morphologiquement lorsque c'est le présent qui est utilisé.

Dans ces exemples, à nouveau, il paraîtrait bien artificiel de calquer l'alternance des temps en anglais; rien ne permet d'identifier une rupture de repérage qui justifierait le passage au présent, et c'est ce que confirment les traductions spontanées proposées par des anglophones, ou les traductions publiées de nombreux autres exemples de ce type. On ne peut donc pas dire que c'est le type de texte (récit véridique par opposition au récit de fiction) qui détermine la possibilité de maintenir en anglais l'hétérogénéité temporelle rencontrée en français. Pourtant, l'observation d'un corpus de textes en anglais fait apparaitre que l'alternance temporelle existe bel et bien dans cette langue aussi. Quelles en sont donc les conditions d'apparition?

\section{Quelques exemples de tense-switching en anglais}

Les exemples (8) à (11), qui font alterner le prétérit et le présent simple dans une même séquence narrative, présentent diverses caractéristiques qui permettent de dire que la rupture temporelle est en quelque sorte préparée, favorisée par d'autres paramètres sémantiques et syntaxiques.

(8) My mother did not allow me to delay going to school just because the FBI had come to the door. [...] The men went inside and, going down the splintery front steps, I turned and caught a glimpse of Paul coming out of the kitchen to meet them just as the door closed.

When the FBI knocks on your door and wants only to ask a few questions, you do not have to consent to be asked questions. [...] You don't have to do anything if you are not subpoenaed or arrested. But you only learn the law as you go along.

"They don't know what they want," Paul says to Rochelle. [...]

"I'm frightened," my mother says. [...]

The next day the same two FBI men come back again, this time in the early evening.

(Doctorow 1971: 119)

Dans l'extrait (8), la première forme de présent ne se situe pas sur le plan du récit chronologique, mais fait intervenir un commentaire d'ordre général, fondé sur l'itération de la relation the FBI knocks qui se trouve repérée non pas par rapport au moment spécifique du récit, mais par rapport à une classe de situations hors plan du récit. Le retour au récit chronologique se fait ensuite par le biais de deux répliques de discours direct cité, avec verbe déclaratif postposé: il y a là un nouveau décrochage, cette fois-ci du plan d'énonciation (récit-discours rapporté) ; le verbe déclaratif postposé ne constitue pas à proprement parler un événement de la trame narrative destiné à introduire les paroles prononcées, mais plutôt une qualification secondaire du discours, qui se contente d'attribuer les paroles à un locuteur donné; de plus, le verbe de parole say est noté comme étant très fréquemment employé au présent dans le récit, même en cas de récit majoritairement au prétérit (cf. Wolfson 1982; Chuquet 
1994). On a donc dans cet extrait toute une série de facteurs «annexes» qui favorisent le passage sans heurts de The men went inside à The next day the same two FBI men come back again. La juxtaposition des deux énoncés serait beaucoup plus difficile, alors qu'en français elle ne poserait sans doute pas de problème.

(9) One episode, more than any other, reveals to us Jeanmaire's state of mind during the high-days of his honeymoon with Denissenko: it is the bizarre encounter on 30 November 1963 when Denissenko called on Jeanmaire in his apartment in Lausanne. According to Jeanmaire the scene unfolded in this way.

Marie-Louise is in the kitchen. Jeanmaire, with his customary ambiguity when speaking of her, no longer remembers whether she is party to the conversation.

Denissenko to Jeanmaire: ... Producing a large envelope, unsealed, he holds it out to Jeanmaire [...] 'It's a compensation,' Denissenko explains.

Jeanmaire takes the envelope and flings it on the floor.

(J. Le Carré, Granta 35, printemps 1991, p. 48-49)

L'extrait (9) présente des points communs avec le précédent, dans la mesure où l'on rencontre à nouveau des énoncés de discours rapporté et des verbes déclaratifs. Le repère-origine de l'assertion rapportée est explicitement posé dans le récit au prétérit à la fin du premier paragraphe: According to Jeanmaire the scene unfolded in this way. Cette phrase force une sorte d'arrêt sur image dans le récit, et annonce une séquence narrative qui n'est plus construite directement en rupture par rapport au repère origine de l'énonciation, mais présentée comme se déroulant sous les yeux d'un observateur présent dans le récit (noter le rôle que joue le déictique: in this way). Le passage du prétérit au présent correspond donc à la rupture entre récit rétrospectif construit à partir du moment de l'énonciation et récit-reportage présenté comme fictivement contemporain d'un observateur rapporté.

(10) Until we moved to Toronto I was happy. Before that we didn't really live anywhere; or we lived in so many places it was hard to remember them. We spent a lot of time driving, in our low-slung, boat-sized Studebaker, over back roads or along two-lane highways up north, curving past lake after lake, hill after hill, with the white lines going down the middle of the road and the telephone poles along the sides, tall ones and shorter ones, the wires looking as if they were moving up and down.

I sit by myself in the back of the car, among the suitcases and the cardboard boxes of food and the coats, and the gassy dry-cleaning smell of the car upholstery. My brother Stephen sits in the front seat, beside the partly open window. [...] Sometimes he throws up into paper bags, or beside the road if my father can stop the car in time.

(Atwood 1990: 21)

(11) Last week, I set off during my lunch hour in search of self-protection [...] I wasn't sure what I was looking for. I was no different from hundreds of other busy working women all over the country.

So I look for self-defence classes in the Yellow Pages; there is nothing listed. I know you can't buy or carry Mace (an irritant gas) or pepper in aerosol cans in Britain [...] Instead I go out to try to buy an attack alarm...

(The Independent, 9 août 1992)

Les extraits (10) et (11) — l'un littéraire, l'autre journalistique — sont représentatifs du type de configuration dans lequel on rencontre le plus facilement l'alternance prétérit - présent en anglais. Il s'agit de récits à la première personne mettant en scène un $I$ soit fictif, dans le roman, soit tout à la fois identique à et différent de 
l'énonciateur dans le récit véridique. Dans les deux cas, le champ du révolu, ou de l'ailleurs de la fiction, est d'abord posé par les énoncés au prétérit, qui marquent la rupture par rapport au moment de l'énonciation; ce marqueur temporel de mise en récit est la trace de la construction du je-personnage par le je-énonciateur origine. Une fois installé ce plan révolu ou fictif, le présent peut apparaître sans problème, soit pour évoquer un souvenir, une scène typique, une sorte d'arrêt sur image (10) ou une séquence d'événements racontés sur le mode du reportage en direct (11).

On voit donc que les ruptures temporelles sont tout à fait possibles dans le récit en anglais, mais qu'elles sont soumises à des contraintes plus fortes que celles que l'on peut rencontrer en français. J'ai essayé de trouver une explication à cette différence en étudiant, dans le cadre de la théorie des opérations énonciatives d'Antoine Culioli ${ }^{4}$, le mode de construction du récit dans chacune des deux langues.

Si l'on admet que l'origine de toute production langagière est la situation d'énonciation origine $\left(S i t_{0}\right)$, repère abstrait muni de deux paramètres, l'un subjectif (sujet énonciateur $S_{0}$ ) et l'autre spatio-temporel (moment et lieu de l'énonciation $T_{0}$ ), l'étude du récit au présent en français et en anglais fait apparaitre que les deux paramètres, subjectif et spatio-temporel, ne s'articulent pas de la même façon dans les deux langues.

Le présent français, interprété par défaut comme étant la marque d'un aspect inaccompli et d'un repérage par identification par rapport au moment de l'énonciation, est en fait une forme très faiblement déterminée du point de vue aspectuel et temporel (voir Serbat 1988). Ce qui le caractérise avant tout, c'est la possibilité d'être repéré par identification par rapport à un repère, quel qu'il soit: il suffit donc d'avoir une date, un repère objectif spatio-temporel qui installe le révolu pour que le présent historique puisse prendre le relais. La rupture sur la composante spatio-temporelle (T) de la situation d'énonciation origine est suffisante pour que le présent fonctionne dans le récit, et n'empêche pas qu'à tout moment il y ait possibilité de retourner sur la construction du récit à partir du repère subjectif $(S)$ de la situation d'énonciation origine, d'où l'extrême souplesse du passage d'un plan à l'autre et, par conséquent, des alternances passé-présent dans le récit.

Le présent simple anglais n'a pas les mêmes caractéristiques aspectuelles que le présent français puisque, s'opposant au présent "progressif», il se définit d'emblée par sa valeur de non-repérage par rapport au moment de l'énonciation (présent de généralité, d'itération, de propriété) et, partant, de non-repérage par rapport à tout autre index temporel. Le présent anglais sert en priorité à prédiquer un procès en tant qu'il caractérise un sujet, et c'est ce sujet qui doit être d'une façon ou d'une autre repéré par rapport à une situation. Le récit au présent en anglais est donc la trace d'une rupture plus radicale par rapport à $S i t_{0}$, qui opère à la fois sur $\mathrm{T}$ et sur $\mathrm{S}$. De ce fait, le va-et-vient entre les deux types de repérage est beaucoup plus difficile à effectuer: une fois situé sur le plan du radicalement «autre», l'anglais y reste, et tous les procès vont être repérés par rapport au point d'observation fictif que l'on est obligé de postuler.

C'est la raison pour laquelle on rencontrera peu — ou pas du tout — le présent en anglais dans les récits écrits d'événements véridiques révolus, récits dans lesquels il n'est pas justifié de poser une rupture sur le paramètre subjectif, puisqu'ils sont intégralement pris en charge et reconstruits rétrospectivement à partir de Sit. $t_{0}$ C'est aussi la raison pour laquelle le récit à la première personne, qu’il soit fictif ou véridi- 
que, est le plus propice à l'emploi du présent et des ruptures temporelles: par définition, le récit à la première personne suppose une rupture fictive sur le paramètre $S$ (le $j e$ du récit est à la fois le même et l'autre par rapport au je-énonciateur) et se prête tout particulièrement aux ruptures de repérage, selon que le je reconstruit son histoire à partir de $S_{i t}$ ou qu'il se place en observateur fictif dans le plan du décroché. C'est ce que l'on peut observer dans le type de récit véridique illustré par (11), où l'énonciateur commence par se poser en personnage par le biais de la rupture temporelle, pour ensuite construire délibérément le décrochage entre le je-énonciateur et le je-repère origine fictif (So I look...). La nécessaire rupture sur le paramètre subjectif en anglais explique aussi pourquoi les changements de locuteurs, de plans d'énonciation avec situation d'assertion rapportée, les transitions effectuées à l'aide de commentaires génériques ou d'énoncés de propriété susceptibles d'être mis sur le compte de l'énonciateur origine servent de «béquilles» à l'hétérogénéité temporelle: si l'on ne peut pas quelque part récupérer de traces d'un décrochage du point de vue subjectif, on ne pourra pas justifier de rupture temporelle à l'intérieur d'une même séquence narrative.

Les remarques qui précèdent devraient nous donner quelques éléments pour guider les choix de traduction du français vers l'anglais, sachant qu'il reste toujours une part de subjectivité, et que tout n'est pas explicable ou justifiable dans ce domaine par des critères strictement linguistiques, loin s'en faut.

\section{Quelques études de cas pour définir des critères de choix de traduction}

Je voudrais donc dans cette dernière partie examiner quelques extraits tirés de types de récits divers pour essayer de voir si, à partir des hypothèses que je viens de résumer, il est possible de trouver des critères fiables sur lesquels se baser pour donner des «consignes» de traduction, sans pour autant prétendre apporter de réponse ferme et définitive.

Je commencerai par deux articles de presse, dont le premier me semble exclure l'hétérogénéité temporelle en anglais alors que le second, au contraire, l'autorise. Pourquoi?

(12) Dimanche, [Khotso Seatlholo] a fait un long travelling arrière sur sa vie et s'est rappelé ce mercredi 16 juin 1976, un peu "comme un mauvais cauchemar». Il s'est remémoré la nuit frénétique précédant la manifestation, lorsque, vice-président du Conseil représentatif des étudiants de Soweto (SSRC), il retrouvait ses amis pour peindre des slogans et préparer secrètement l'événement du lendemain. [...]

Ce «mercredi noir», la manifestation des écoliers de Soweto tourne à l'hécatombe. Le premier à tomber est un écolier de 13 ans, Hector Petersen...

(Libération, 17 juin 1996)

(13) Le soir tombe. Au pied de l'hôtel de ville de Poitiers, des vaches blondes paissent dans un enclos de fortune comme si elles avaient toujours été là. Autour, c'est la fête. On se régale de viande grillée et de mijet (soupe de pain et de vin). Les organisateurs de la «marche sur Paris» sont un peu débordés par le succès. [...]

Au départ, c'est une idée un peu folle. Un pari lancé il y a un mois entre une poignée de copains de Charroux, dans le sud de la Vienne. "Ce qu'il faut faire, c'est amener cent vaches à Paris», a dit l'un. Les autres ont rigolé. Puis réfléchi.

(Le Monde, 15 août 1996) 
En (12) le caractère de rétrospective historique d'événements véridiques (vingtième anniversaire des émeutes de Soweto) ainsi que l'antéposition du repère temporel objectif situé dans le révolu ce mercredi noir, contraint à l'emploi d'une forme marquant la rupture temporelle, à savoir le prétérit: On that "black Wednesday," the Soweto students' demonstration turned into a massacre. Malgré la présence de citations du personnage autour duquel est organisé l'article, il n'y a pas, au niveau de la rétrospective des événements d'un point de vue factuel, de changement de plan énonciatif, donc rien ne justifie l'alternance en anglais. En revanche en (13) le premier paragraphe de «mise en scène» de cet article de reportage me semble pouvoir, et même devoir être traduit au présent, car il y est brossé un tableau actualisé de la scène, avant le démarrage proprement dit du récit. Le décrochage du point de vue subjectif a bien lieu, les événements se déroulent sous les yeux d'un observateur fictif (façon de «faire entrer» le lecteur dans le vif du sujet), et c'est le présent progressif d'identification à un point de vue interne à la situation qui sera choisi (Night is falling... cows are grazing...). Cependant, la divergence entre le français et l'anglais apparaît au second paragraphe. L'énoncé Au départ, c'est une idée un peu folle illustre bien la capacité du présent français à «jouer sur les deux tableaux» - à la fois présent historique repéré par rapport au repère de localisation temporelle situé dans le révolu et commentaire de l'énonciateur situé en $T_{0}$. Cette composition de repérages n'étant pas possible en anglais, c'est la localisation dans le révolu, donnée par le circonstant de temps, qui prend le dessus, et contraint à passer au prétérit dès cette première phrase du paragraphe (It all started with a rather crazy idea), sans attendre le début du récit des événements, effectué en français au passé composé, après la première réplique de discours rapporté.

L'extrait suivant est tiré du roman de Jean Rouaud, Les Champs d'honneur, qui fait un usage abondant des ruptures temporelles, dont certaines sont d'ailleurs déroutantes, même en français.

(14) C’est vrai qu'ils n'étaient plus jeunes. Ils en convenaient encore à la descente du train, déposant sur le quai quatre lourdes valises, grand-père s'épongeant le front sous son panama, grand-mère s'éventant avec le journal mal replié de la veille, fourbus, le visage souligné de fines nervures sombres contractées sous le panache fuligineux de la locomotive - et, tandis que John, le mari anglais de Lucie, empoigne les valises et les dépose sur un chariot, que le petit groupe s'éloigne du pas méticuleux des vieux parents vers le ciel impeccablement bleu de la sortie, on débat, au vu de leur fatigue, s'il ne serait pas mieux de passer par Lyon...

(Rouaud 1990: 39)

Dans cet exemple, la transition du passé au présent est cependant tout à fait classique, intervenant dans une subordonnée temporelle qui marque une rupture dans la séquence des événements et déclenche un changement de localisation spatiale. Je ne pense pas que l'on puisse justifier une rupture semblable en anglais: le récit est à la troisième personne, il n'y a pas de rupture manifeste d'origine du point de vue, donc pas de raison d'employer autre chose que le prétérit dans l'ensemble du paragraphe. C'est ce choix qu'a fait le traducteur du roman, Ralph Manheim, qui maintient le prétérit pour while John, Lucie's English husband, picked up the suitcases and carried them... Pour approfondir l'analyse, il aurait fallu reproduire plusieurs pages du roman. En effet, tout au long de l'œuvre, le traducteur est attentif à ne pas banaliser le texte français, tout en obéissant aux contraintes narratives de l'anglais: 
ici, il attend la fin de la page suivante pour passer du prétérit au présent en anglais, à la faveur d'une séquence de discours indirect libre qui permet, grâce au changement de plan d'énonciation, de "préparer le terrain» pour la rupture temporelle.

Voici maintenant quelques exemples où, me semble-t-il, l'hétérogénéité temporelle peut fonctionner en anglais quoique, comme on peut le constater, tous les traducteurs ne s'y hasardent pas.

(15) Il sortit un grand mouchoir dont il s'épongea le front et je me rappelle brusquement que ce geste lui est familier. Il se tait. Moi aussi. La lumière baisse. Là-bas, les trois autres tiennent un conciliabule.

(Modiano 1972: 51)

He took out a large handkerchief and wiped his forehead, and I remember that this is a favourite gesture of his. He is silent. So am I. It's getting darker. Over there the other two are talking in low voices.

(16) À l'instant où [le métro] débouchait, on m’a poussé brutalement dans le dos.

Ensuite, je suis allongé sur l'un des bancs de la station. Un petit groupe de curieux m'entoure $[\ldots]$ Deux gardiens de la paix m'aident à me relever. Ils me soutiennent par les aisselles. Nous traversons la station.

(Modiano 1972: 99)

Just as [the train] came into sight, someone pushed me roughly in the back.

Afterwards, I find myself lying on one of the station benches. A little group of curious people surrounds me [...] Two policemen help me stand up. Support me under the arms. We go across the station.

(17) A la descente du wagon, Thérèse ne fut pas reconnue. Pendant que Balion remettait son billet, elle avait contourné la gare et, à travers les planches empilées, rejoint la route où stationnait la carriole.

Cette carriole, maintenant, lui est un refuge; sur le chemin défoncé, elle ne redoute plus de rencontrer personne.

(Mauriac 1927: 119)

Thérèse was not recognized as she left the train. While Balion was giving up her ticket she walked round to the other side of the station and made her way between the wood-stacks to the road, where the trap was waiting.

It had become, for her, a refuge. There was small risk that anyone would meet them in the unmetalled lane.

Les deux extraits de Modiano - (15) et (16) - font partie de ceux qui, dans la traduction calquée qu'en propose C. Hillier, passent assez naturellement en anglais, ce qui n'est pas toujours le cas dans cette traduction. Dans les deux cas intervient la mise en scène d'un souvenir, dans le cadre d'un récit à narrateur homodiégétique, permettant de postuler le décrochage dans le récit d'un repère-origine du point de vue. C'est d'ailleurs par rapport à ce point de vue que sont repérées les formes aspectuelles de présent progressif en anglais, produisant un effet plus marqué de description, d'arrêt sur image, par contraste avec la neutralité aspectuelle, et par conséquent l'ambiguiité entre description et narration, du présent français. La mise en place du souvenir est explicite dans les deux langues en (15): je me rappelle / I remember; en revanche en (16), alors que le français peut juxtaposer sans autre marqueur de décrochage que l'adverbe temporel antéposé l'énoncé au passé composé: on m’a poussé et l'énoncé au présent: Ensuite, je suis allongé, l'anglais semble contraint 
d'expliciter le repérage du second par rapport à une origine du point de vue décrochée dans le récit: I find myself..., faute de quoi la séquence paraîtrait incohérente.

L'extrait (17), tiré de Thérèse Desqueyroux, ne me semble pas foncièrement différent des précédents en ce qui concerne la possibilité de l'alternance temporelle en anglais. En effet, le passage du passé simple au présent en français s'accompagne d'une rupture de point de vue, explicitement signalée par l'adverbe maintenant ${ }^{5}$. Cette rupture est totalement gommée dans la traduction anglaise, qui a fait le choix de la «naturalisation» par le recours homogène au prétérit. On ne peut s'empêcher d'y voir une perte, comme dans bien des traductions de cette période de l'entredeux-guerres - voir aussi les traductions de Malraux par Stuart Gilbert et Alasdair McDonald, ou encore celles de Flaubert par Gerard Hopkins et J. Lewis May (citées in Guillemin-Flescher 1981) — qui cherchaient à ne pas heurter les sensibilités linguistiques du public anglophone et à occulter toute étrangeté de l'original (voir sur ce problème les écrits de Berman, en particulier 1984). Ne pourrait-on pas envisager de conserver l'effet de rupture en anglais, et de traduire par un présent: Now she feels that the trap is a refuge for her; she is no longer fearful of...

Quels conseils donner, enfin, pour la traduction des deux extraits suivants?

(18) La chambre était tendue de soie bleu pâle. [...] La lampe brillait doucement et la flamme du foyer faisait palpiter comme des des ailes dans l'ombre. Assise en robe de chambre devant le bonheur-du-jour, son cou délicat incliné sous la magnifique et pâle auréole de ses cheveux, Julie feuillette les lettres qui dormaient, liées avec des faveurs dans les tiroirs du meuble.

Minuit sonne; c'est le signe du passage d'une année à l'autre. La mignonne pendule, où rit un amour doré, annonce que l'année 1793 est finie.

Au moment de la conjonction des aiguilles, un petit fantôme a paru. Un joli enfant, sorti du cabinet où il couche et dont la porte reste entrouverte, est venu, en chemise, se jeter dans les bras de sa mère et lui souhaiter une bonne année.

(France 1892)

(19) Simm se hâta vers l'emplacement où il avait groupé, en un petit tas, les restes du volet bleu.

Dès la première secousse - il s'en souvenait de plus en plus distinctement - ce morceau, qui formait l'angle de la façade, s'était séparé, détaché, de l'ensemble, avait glissé en avant, entraînant la fenêtre et le chambre du jeune homme dans sa chute, évitant - de quelques secondes - l'écrasement final sous l'énorme dalle de la terrasse.

Le vieillard s'agenouille, recueille par poignées le ramassis de poussière, de cailloux; le jette au loin, cherchant à dégager l'orifice. Ensuite, pour ne plus perdre celui-ci de vue, il décide de singulariser l'endroit en dressant autour une petite enceinte. Ainsi pourra-t-il, sans difficulté et d'où qu'il se trouve sur le terrain, le repérer, ou indiquer le lieu exact aux autres, dès que ceux-ci reparaîtront.

Forcément, ils reviendront.

(Chédid 1969: 55)

Le caractère classique de la narration d'Anatole France en (18), dont le cadre est posé à l'imparfait et le personnage vu exclusivement de l'extérieur me feraient pencher pour un emploi homogène du prétérit en anglais. Si une rupture temporelle est possible (ce dont je ne suis pas convaincue), elle ne pourrait intervenir que sur l'événement perturbateur: Minuit sonne - The clock strikes midnight, et non dans la phrase précédente, et contraindrait à poursuivre le récit au présent, afin de ne pas verser dans l'incohérence. En revanche, il me semblerait dommage d'introduire une 
telle distance énonciative en traduisant l'ensemble du récit d'Andrée Chédid (19) au prétérit, dans la mesure où Simm le vieillard, quoiqu'étant un personnage désigné par la troisième personne, est véritablement le centre de conscience de cette scène voir le verbe de souvenir, et le discours direct libre de la dernière phrase. Je serais par conséquent encline à préserver la rupture temporelle prétérit - présent, en l'annonçant dès l'apparition du verbe de souvenir, qui permet de construire le personnage en repère-origine fictif dans le récit (donc: he can remember), et éventuellement en réactualisant ce repère privilégié par l'insertion de l'adverbe démarcatif now au début du troisième paragraphe: Now the old man kneels down...

Les remarques qui précèdent sur ces quelques extraits sont loin de résoudre tous les problèmes, mais il me semble tout de même que grâce à l'observation de corpus dans les deux langues et aux hypothèses avancées sur le mode de construction du récit par rupture en français et en anglais, on peut être un petit peu moins démuni pour expliquer et justifier les choix qui s'offrent aux traducteurs.

L'identification du type de récit est l'opération préliminaire indispensable à tout choix, face à cette question de l'alternance temporelle. Dans le cas d'un récit d'événements véridiques, s'il ne comporte aucune trace de décrochage d'un point de vue subjectif, de mise en scène, de velléité de présentation d'un récit «sur le vif», on penchera en anglais pour l'homogénéité temporelle dans le cadre du passé. Il faut savoir reconnaitre que la rhétorique des alternances temporelles dans le récit journalistique et historique en français est en passe de se banaliser pour devenir une nouvelle norme narrative, et il ne faut pas voir dans tout emploi du présent dit historique une intention marquée de mise en relief ou d'actualisation.

Dans le cas de récits littéraires relevant de la fiction, les choses sont plus complexes. La langue d'une œuvre littéraire est le produit de choix énonciatifs plus originaux, plus marqués que celle des échanges ordinaires, et les moyens linguistiques mis en œuvre pour atteindre l'effet souhaité peuvent diverger considérablement par rapport aux usages standard de la langue. C'est là que les réflexions sur le statut du traducteur comme écrivain et sur le processus de traduction comme ré-énonciation prennent tout leur sens, et il est bien difficile d'affirmer à l'apprenti-traducteur ce qui est possible ou impossible, et même d'en être absolument certain soi-même. Ce que j'ai essayé de faire à partir de mes observations, c'est d'identifier un certain nombre de critères relativement objectifs et stables permettant de définir les cas où il est sans conteste possible de maintenir l'hétérogénéité temporelle en passant du français à l'anglais, en ayant recours à des justifications autres que la simple intuition. Il n'en reste pas moins que les jugements de plus ou moins grande acceptabilité que j'ai avancés sont sans aucun doute ouverts à la critique, et que c'est un domaine dans lequel demeurent encore bien des points d'ombre.

\section{NOTES}

1. Voir, par exemple, G. Hardin et C. Picot (1990: 143): «En général, le présent de narration n'existe pas $[s i c]$ en anglais et c'est le prétérit qu'on doit utiliser pour traduire le présent de narration français.»

2. Voir en particulier l'étude très complète effectuée par Casparis (1975) sur les emplois du présent historique dans les récits littéraires en anglais.

3. Sur le rôle de l'imparfait dans le récit au présent, voir notamment Boyer (1985), Imbs (1960) et Labelle (1987). 
4. Voir Culioli (1990), Fuchs et Léonard (1979) pour une présentation générale de la théorie et l'application de ses concepts à l'étude des phénomènes aspectuels; voir plus particulièrement Culioli (1978) sur le type de repérage aoristique à l'œuvre dans le récit au présent historique.

5. Voir Jouve (1992) sur les rapports entre l'adverbe déictique maintenant et les ruptures temporelles, dans son analyse du roman Au Château d'Argol de Julien Gracq.

\section{RÉFÉRENCES}

a. Sources du corpus - ouvrages traduits

Atwood, Margaret (1990): Cat's Eye, Virago.

BLoch, Marc (1939): La société féodale, t. 1 (La formation des liens de dépendance), Paris, Albin Michel (réimpr. 1989); trad. ang. L. A. Manyon (1961): Feudal Society, Londres, Routledge and Kegan Paul (réimpr. 1967).

Chahine (1980): Le Livre de Daniel, Paris, Robert Laffont, coll. «10-18».

ChÉdid, Andrée (1969): L’Autre, Paris, Flammarion.

DaEninckx, Didier (1984) : Le géant inachevé, Paris, Gallimard, coll. "Série Noire».

Doctorow, E. L. (1971): The Book of Daniel, Random House (réimpr. 1979, Bantam); trad. fr. Gilles.

France, Anatole (1892): «La perquisition», Nouvelles révolutionnaires, Paris, Presses Pocket.

Mauriac, François (1927): Thérèse Desqueyroux, Paris, Grasset, coll. "Le Livre de Poche», $\mathrm{n}^{\circ} 138$; trad. ang. Gerard Hopkins (1947): Thérèse, Londres, Eyre and Spottiswoode (réimpr. 1959, Penguin Books).

Modiano, Patrick (1972): Les boulevards de ceinture, Paris, Gallimard, coll. «Folio»; trad. ang. Catherine Hillier (1974): Ring Roads, Londres, Victor Gollancz.

— (1984): Quartier perdu, Paris, Gallimard; trad. ang. Anthea Bell (1988): A Trace of Malice, Henley-on-Thames, Aidan Ellis Publishers.

Prassinos, G. (1972) : «La gouvernante», La lucarne: Nouvelles, Paris, Gallimard.

Rouaud, Jean (1990): Les Champs d'honneur, Paris, Éditions de Minuit; trad. ang. Ralph Manheim (1992): Fields of Glory, Harvill.

YourCEnAR, Marguerite (1977): Archives du Nord, Paris, Gallimard.

\section{b. Études}

Bellos, D. (1978) : «The Narrative Absolute Tense», Language and Style, 11-4, p. 231-237.

Berman, A. (1984) : L'épreuve de l'étranger, Paris, Gallimard.

Boyer, H. (1985) : «Le temps dans la mise en scène du récit de vie», Pratiques, 45, p. 52-64.

Casparis, C. P. (1975) : Tense without Time. The Present Tense in Narration, Berne, Francke, coll. «Swiss Studies in English».

Chuquet, H. (1994): Le présent de narration en anglais et en français. Linguistique contrastive et traduction, numéro spécial, Gap, Ophrys.

Comrie, B. (1976) : Aspect, Cambridge, Cambridge University Press.

Culioli, A. (1978): "Valeurs aspectuelles et opérations énonciatives: l'aoristique», Actes du Colloque sur la notion d'aspect (J. David et R. Martin, dir.), Publication de l'Université de Metz, p. 181-193.

— (1990): Pour une linguistique de l'énonciation. Opérations et représentations, Gap, Ophrys.

Dolz, J. (1993): «Bases et ruptures temporelles: étude de l'hétérogénéité temporelle des esquisses biographiques", Langue française, 97, p. 60-80.

Fleischman, S. (1990): Tense and Narrativity. From Medieval Performance to Modern Fiction, Londres, Routledge.

Fuchs, C. et A. M. LÉONARD (1979): Vers une théorie des aspects. Les systèmes du français et de l'anglais, Paris, Mouton.

Guillemin-Flescher, J. (1981): Syntaxe comparée du français et de l'anglais. Problèmes de traduction, Gap, Ophrys. 
Hardin, G. et C. Picot (1990): Translate. Initiation à la pratique de la traduction, Paris, Dunod. IмBs, P. (1960): L'emploi des temps verbaux en français moderne: essai de grammaire descriptive, Paris, Klincksieck.

Jouve, D. (1992): "'Maintenant' et la deixis temporelle», La Deixis (L. Danon-Boileau et M. A. Morel, dir.), Paris, Presses universitaires de France, p. 355-363.

Labelle, M. (1987): «L'utilisation des temps du passé dans les narrations françaises: le Passé Composé, l'Imparfait et le Présent Historique», Revue Romane, 22-1, p. 3-29.

Monville-Burston, M. et L. Waugh (1985) : «Le passé simple dans le discours journalistique », Lingua, 67, p. 121-170.

SChiffrin, D. (1981): «Tense Variation in Narrative», Language, 57-1, p. 45-62.

Serbat, G. (1988): «Le prétendu "présent" de l'indicatif: une forme non déictique du verbe», L'information grammaticale, 38, p. 32-35.

Simonin, J. (1984) : «Les repérages énonciatifs dans les textes de presse», La langue au ras du texte (A. Grésillon et J. L. Lebrave, dir.), Lille, Presses universitaires, p. 133-203.

Wolfson, N. (1982): The Conversational Historical Present (CHP) in American English Narrative, Dordrecht, Foris Publications. 